\title{
Peertechz
}

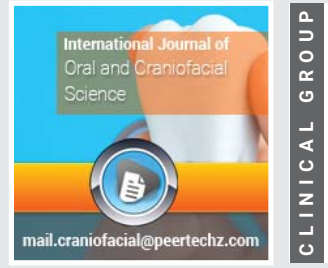

Research Article

\section{The comparative assessment of the of the effectiveness of} \section{immediate and delayed dental}

\section{implantation}

\section{Gagik Hakobyan'*, Lazar Esayan², Davit Hakobyan³ , Gagik Khachatryan ${ }^{4}$ and Gegham Tunyan ${ }^{5}$}

${ }^{1}$ Professor, Head of Department of Oral and Maxillofacial Surgery Yerevan State Medical University

after M. Heratsi, Armenia

${ }^{2}$ Professor, Head of Department of Therapeutik Stomatology Yerevan State Medical University after M.

Heratsi, Head of the Department of Maxillofacial Surgery of Medical Center MIM, Armenia

${ }^{3}$ Assistant professor, Department of Oral and Maxillofacial Surgery, Yerevan State Medical University

after M. Heratsi, Armenia

${ }^{4}$ Head of Department postgraduate students Dentistry

${ }^{5}$ Dental Resident at Yerevan State Medical University after M. Heratsi, Armenia
Received: 19 June, 2020

Accepted: 09 November, 2020

Published: 18 November, 2020

*Corresponding author: Gagik Hakobyan, Professor, DMSc,PhD, Mailing addres: 0028 Kievyan str. 10 ap. 65 Yerevan, Armenia, Tel: (+37410)271146;

E-mail:prom_hg@yahoo.com

Keywords: Immediate dental implantation; Delayed dental implantation

https://www.peertechz.com

\section{Check for updates}

\section{Abstract}

Objective: The purpose of this study was to evaluate the outcome of immediate and delayed implant placment protocols.

Materials and methods: The 52 patients with missing thoot (from 2014 to 2019) were selected for the study, total 64 implants were placed. All patients presented functional and esthetic complaints and underwent a thorough clinical examination according to a generally accepted scheme. Computed tomography were obtained to determine the osseos structure.

To conduct a comparative analysis of the treatments results, two groups were formed:

-Basic group of 28 patients -were placed 36 immediate implants.

-Control group of 24 patients-3-5 months after extraction of tooth were placed 28 delayed implants.

Postoperative outcomes; infection, radio-density, resorption and failure of implants were checked clinically and radiographically using serial orthopantomograms or CT scan.

The implants stability were evaluated with measures of resonance frequency analysis (RFA) during the follow-up periods using Osstell Mentor at time of implant placement, after 3-6 months. The functional load on dental implants was performed with ISQ values above 65 . Dental prosthetic rehabilitation was performed after 3-6 months of submerged healing in 35 patients. Early dental prosthetic loaded in 17 patients ( 9 patients in basic group and 8 control group).

Results: No serious intraoperative or immediate postoperative complications were noted. After a 24-month follow-up period, the basic group resulted in a mean bone loss of 1.04 and the control group of $1.02 \mathrm{~mm}$, there were no statistically significant differences. Clinical comparing delayed and immediate implant placement there were no statistically significant differences effect on soft tissue recession outcomes.

Clinical outcome of implants immediately placed into extraction sockets of teeth affected by chronic lesions was examined. 11 Patients with periapical infection and 
17 patients without it for immediate placement were chosen. No significant differences were found with periapical infection and without in the basic group patients, no signs of infection around the implants were detected at any control visit.

The survival rate of early-loaded implants placed in extraction sockets demonstrated no implants failures. There are no significant differences in implant stability between immediate and delayed implants. Immediately placed implants were included with an initial primary stability over 65 ISQ and 71.1 ISQ delayed implants. The differences in these results were not statistically significant.

Success rate of immediately placed implants 5 years after was $97,8 \%$ and delayed implants $98,1 \%$. The survival rate of early-loaded implants placed in extraction sockets $96,2 \%$

Conclusion: There are no significant differences in immediate and delayed implants. After dental implant prosthetic rehabilitation, the masticatory function, esthetics of the facial profile and occlusion was improved.

\section{Introduction}

The loss of tooth can occur for various reasons, which include trauma, diseases of the dentition (caries or or periodontal disease). It may also occur secondarily or simultaneously with various systemic diseases such as cancer, diabetes mellitus, osteoporosis etc [1-3].

Due to tooth loss, not only functional impairment occurs in the maxillofacial region, changes in the esthetics of the patient's appearance, but also violates the psychological status of a patients and discomfort occurs during communication [4].

Dental implants are now commonly used for replacing missing teeth in various clinical situations. Conventional procedure for implant placement involves extraction of offending tooth, waiting 3-4 months for extraction socket to heal, insertion of implant, and again waiting for 3-6 months for integration of implant with surrounding bone; after this procedure, another surgery is necessary to expose the implant and to place a prosthetic abutmentand crown [5].The patient had to wait up to 6-8 months for a lost tooth to be replaced.

To substantially shorten the entire treatment were developed by placement of implant immediately after extraction of tooth. In the modern era, immediate placement of a dental implant concept is gaining popularity, especially for anterior teeth. Immediate placement of a dental implant in an extraction socket was initially described more than 30 years ago by Schulte and Heimke in 1976 [6].

Immediate implant placement is most commonly indicated when tooth extraction is due to trauma, endodontic lesion, root fracture, root resorption, root perforation, unfavourable crown to root ratio (not due to periodontal loss) and bony walls of alveolus are still intact. Contraindications includes presence of active infection, insufficient bone $(<3 \mathrm{~mm})$ beyond the tooth socket apex for initial implant stability and wide and/or long gingival recession [7].

Reductions in the number of surgical interventions, a shorter treatment time, the presumptive preservation of alveolar bone of the tooth extraction and soft tissue aesthetics have been claimed as the potential advantages of this treatment approach [8]. Also use of bone graft of materials has been shown to result in predictable regenerate, high levels of osseointegration. Several reviews reported that the immediate implant treatment using autogenous bone grafts or xenografts may improve the process of bone formation between the implant and the surrounding socket walls as well as survival rates [9].
Different placement and loading protocols have evolved from the first protocols in order to achieve quicker and easier surgical treatment times. Now immediately placed and immediate loading implants are more predictable and successful than before [10]. However, this approach cannot be applied to every immediate implant patient. In comparison to conventional implant treatment, the ideal state for immediately loaded implants would include adequate bone quality (D2 bone), and minimum implant length of $10 \mathrm{~mm}$, adequate primary stability and avoidance of lateral forces [11].

Primary stability of immediately placed implant seems to be the most important factor in immediate loading. Quirynen, et al. concluded that the incidence of implant failure is significantly higher when combining immediate implant insertion with immediate loading [12]. Ferrara, et al. conducted a study combining immediate placement and early loading of 33 implants and they found satisfactory esthetic and functional results [13].

Despite the many publications on this topic, the choice of an optimal treatment plan in especially after tooth extraction in the esthetics zone of the jaw is an urgent problem of modern implant dentistry.

The purpose of this study was to evaluate the outcome of immediate and delayed implant placment protocols.

\section{Materials and methods}

The 52 patients with missing thoot were selected for the study. The ages of the patients ranged between 26 and 43 years (24 males and 28females). Duration of study from 2016 to 2020 at the university clinic in the Department of oral and maxillo facial surgery YSMU All patients had a partially or totally edentulous and presented functional and esthetic complaints. The study was reviewed and approved by the Ethics Committee of the of the Yerevan State Medical University after M. Heratsi (protocol N16, 5.10.17) and in accordance with those of the World Medical Association and the Helsinki Declaration. Informed consent patients were informed verbally and in writing about the study and gave written informed consent.

Patients underwent a thorough clinical examination according to a generally accepted scheme. Preoperative planning includes a careful history and physical exam, in addition to preoperative radiologic investigation, which could include orthopantomogram and/or a computed tomography scan to evaluate for and rule out any contraindication to implant procedure. Computed tomography were obtained to determine 
the osseos structure. Data obtained from CT scan procedure can view the virtual 3D model from different angles using the software to customize the treatment plan. Clinical studies took into account: localization of the defect, the presence of the inflammatory process and the volume of bone tissue in the area of surgical intervention, a combination of direct implantation with other surgical interventions. All patients signed an informed consent for surgery and participation in scientific studies.

To conduct a comparative analysis of the results, two groups were formed:

-Basic group of 28 patients -were placed 36 immediate implants.

-Control group of 24 patients 3-5 months after extraction of tooth were placed 28 delayed implants.

Total 64 implants Ankylos (Dentsply Implants, Germany) dental implants were installed the diameter of the implants used was 3.75 or $4.2 \mathrm{~mm}$ in a variety of lengths (10 to $13 \mathrm{~mm}$ ), depending on the bony morphology.

The helical conical shape of the Ankilos dental implant weth modified surface is shows is optimal in terms of providing primary stability, and the implant to perform the function of osteocondensers and optimally fixed in bone tissue.

Based on the clinical picture and radiological data, we have established the following indications for immediate implantation:

1. Tooth injury - dislocation and inappropriateness of its reduction.

2. Fracture in the middle or upper third of the tooth root, especially with an offset.

3. Tooth decay with poorly sealed canals, with remnants of pins or endodontic instruments, the restoration of which is impossible.

Immediate implantation was performed after the completion of bone growth, that is, at the age of over 18-20 years.

Immediate implantation was performed in different clinical situations; upper or lower jaw, anterior or posterior sites, implants with or without guided bone regeneration, and with or without periapical pathology. 16 patients basic group did not require any type of regenerative procedure (no defect), 12 were filled with biomaterials ( 4 is this patients were filled with biomaterials and also had a resorbable membrane).

The implantation was carried out with local injection anesthesia, the addition of a vasoconstrictor and its percentage were determined according to indications. The removal of teeth was carried out according to the rules adopted in surgical dentistry, and was carried out by, providing for minimal trauma to the alveoli and its surrounding bones. The excess and inflammatory changed mucous membrane that has grown into the cavity of the root of the tooth was removed, if there were granulations on the inner surface of the gingival margin, they were scraped. Degranulation of the socket was immediately performed after dental extraction. The bone socket well was washed with a chlorhexidine solution. The choice of implant should be based on the following criteria: exceed the size of the hole of the extracted tooth by $2-4 \mathrm{~mm}$ in length and 1-2 $\mathrm{mm}$ in width. Choosing an implant from those that were selected before the operation, we checked the size of the depth of the hole and determined the possible bone size for the implant to be inserted into the bone using X-ray data. Bone drilling for implant placement was performed with a guide and forming drill with external cooling of $0.9 \%$ sodium chloride solution.

With insufficient bone size, more often from the vestibular side, in the area of the central teeth, the formation of the socket was carried out the palatine side on the upper jaw and lingual on the lower jaw. The delivered implant must be tightly fixed in the bone, if there were voids in the alveolus, they were filled with osteoplastic materials, autologous bone particls. When using osteoplastic materials in the implants cervical region, the wound was reliably isolated. With a thin cortical plate near the bottom of the nose, drilling in the bones to deepen the implant was carried out distally from the upper point of the cortical plate, but maintaining the size of the bone between the tip of the implant at least $1-3 \mathrm{~mm}$

After checking the length and sufficiency of the soft tissues to close the wound, the absence of tension, we proceeded to the suturing of the soft tissues. At high tension, a laxative incision was made $1 \mathrm{~cm}$ long at the transitional fold. The mucoperiosteal flap was placed in place, sutured with polyamide thread or thin silk. After the operation, cold was applied for 15 to 20 minutes.

The implants stability were evaluated with measures of resonance frequency analysis (RFA) during the follow-up periods using Osstell Mentor at time of implant placement, after 3-6 months.

Dressings were carried out 2-3 times within 10 days, then examined the patient on the 21st day. Cases in which the area to be rehabilitated in the dental arch had adequate bone height and width and a favorable gingival phenotype, implant immediately loaded in fresh sockets after tooth extraction. Before installing the final crowns, temporary crowns were used to ensure adequate gingival contour.

When a periapical infection was present the implant was not placed immediately, instead a delayed placement protocol was performed. When infection was present, granulation tissue was removed previously and antibiotics were given (Amoxicillin $750 \mathrm{mg} 1 \mathrm{~h}$ before the treatment and 750mg every 8hours, 5 days post-operation).

Postoperative outcomes; implant stability, immediate implant survival and success rates, infection, radiodensity, marginal bone loss, failure of implants were checked clinically and radiographically using serial orthopantomograms or CT scan.

After excision of the soft tissues, the cover screw was removed, the toilet of the internal part of the implant was diluted with a chlorhexidine solution, and a healing former 
was fixed, which remained fixed for 2 weeks. The functional load on dental implants was performed with ISQ values above 65. Before installing the final crowns, temporary crowns were used to ensure adequate gingival contour. Dental prosthetic rehabilitation was performed after 3-6 months of submerged healing in 33 patients. Early dental prosthetic loaded in 19 patients (11patients in basic group and 8 control group).

12 patients of basic group implants insertion and dental prosthetic rehabilitation was undertaken immediate after implantation.

16 patients of basic group dental prosthetic rehabilitation was undertaken after 3-6 months implantation.

13 patients of control group implants insertion and dental prosthetic rehabilitation was undertaken immediate after implantation.

11 patients of control group dental implants insertion and submerged healing, prosthetic rehabilitation was undertaken 3-6 months after implantation (patients who needed immediate placement and restoration following extraction of a single tooth).

The criteria for evaluating the effectiveness of treatment were the results of clinical and radiological research methods obtained at the observation stages (quality of the primary fixation of the implant in the fresh sockets of the extracted tooth, the need and amount of use of osteoplastic materials, autologous bones, membrane techniques, terms of healing of the surgical wound, data of $\mathrm{x}$-ray control, terms of prosthetics, functional and aesthetic indicators).

Clinical and radiographic controls were made regularly, the criteria for implant success were assessed. Criteria for failure included: implant mobility ( $>1 \mathrm{~mm}$ ), radiographic bone loss (> 1/3 implant height)

\section{Statistical analysis}

Statistics were used to calculate and analyze the mean marginal bone loss of implants. The differences between follow-up periods were tested by paired Student's t test. All analyses were carried out using SPSS (SPSS Software Company, Chicago, IL, USA). p values $<0.05$ were considered statistically significant.

\section{Results}

No serious intraoperative or immediate postoperative complications were noted. At the control examination after 6 months after surgery, an x-ray examination we did not observe any clinical or radiological signs of inflammation in the area of osteotomy sites and implants.

To evaluate the effectiveness and comparison immediate and delayed implantation methods the following parameters were compared

- Mean marginal bone loss between immediate and delayed implants,
- Differences in implant stability between immediate and delayed implantation.

- Comparing delayed and immediate implant placement effect on soft tissue recession outcomes.

After a 24-month follow-up period, the basic group resulted in a mean marginal bone loss of 1.06 and the control group mean marginal bone loss of $1.02 \mathrm{~mm}$, there were no statistically significant differences (Table 1).

Primary stability results for immediate implants were greater than 65,2 ISQ and 68.3 ISQ delayed implants, measured with the Ostell Mentor. There are no significant differences in implant stability between immediate and delayed implants (Table 2).

\begin{tabular}{|c|c|c|}
\hline \multicolumn{2}{|c|}{ Table 1: The mean marginal bone loss of implants after 24-month. } \\
\hline $\begin{array}{c}\text { The mean crestal } \\
\text { bone loss }\end{array}$ & $\begin{array}{c}\text { Number of basic group } \\
\text { implants }(n=64)\end{array}$ & $\begin{array}{c}\text { Number control group } \\
\text { implants }(n=56)\end{array}$ \\
\hline & $1.06 \mathrm{~mm} \pm 0.25$ & $1.02 \mathrm{~mm} \pm 0.29$
\end{tabular}

Table 2: The Primary stability results for implants.

\begin{tabular}{c|c|c|}
\hline $\begin{array}{c}\text { Stability results for } \\
\text { implants }\end{array}$ & Basic group implants & Control group implants \\
\cline { 2 - 2 } & $65,2 \mathrm{ISQ}$ & $68.3 \mathrm{ISQ}$ \\
\hline
\end{tabular}

Clinical trial comparing delayed and immediate implant placement there were no statistically significant differences effect on soft tissue recession outcomes.

Clinical outcome of implants immediately placed into extraction sockets of teeth affected by chronic lesions was examined. 9 Patients with periapical infection and 17 patients without it for immediate placement were chosen. No significant differences were found with periapical infection and without in the basic group patients, no signs of infection around the implants were detected at any control visit.

Success rate of immediately placed implants 5 years after was $97,8 \%$ and delayed implants $98,1 \%$.The survival rate of early-loaded implants placed in extraction sockets $96,2 \%$.

\section{Case report 1}

A 34-year-old famle patient was reported to the Department of Oral Surgery, with complaints of pain and light mobility of 12 tooth for 3 months. Clinical examination revealed in tooth 12 bleeding on probing, gingival recession, and grade I mobility. Radiographic examination of tooth 12 revealed poor prognosis. (Figure 1A,B).

The patient was aware of the poor condition of tooth. She was very concerned about her esthetics and was willing for procedure of immediate implant with immediate loading. Treatment plan included extraction of tooth 12 , and immediate placement of implant with immediate loading by temporary crown.

Presurgical radiographic evaluation was done and appropriate length and width of available bone were determined 
and dental implant were selected for insertion. Patient was premedicated with $2 \mathrm{~g}$ amoxicillin, $1 \mathrm{~h}$ before surgery. Following injection of $4 \%$ articaine local anesthetic solution, the tooth were atraumatically removed (Figure 1C). Extraction socket were thoroughly debrided and inspected with the help of periodontal probe for any defect or possible perforation of cortical plate. Osteotomy sites were prepared with sequential drills, a more palatal positioning of the implants allows a better primary stability in addition to buccal bone preservation. The implants $(3.75 \times 13 \mathrm{~mm})$, were inserted in the prepared osteotomy site with insertion torque of $40 \mathrm{~N} / \mathrm{cm}^{2}$, and adequate primary stability was obtained. After that stage the biomaterial was placed in the labial gap region between the implant and bone, because the space between implant and bone, when the implant was placed, was greater than $2 \mathrm{~mm}$.Periodontal tissue was preserved, respecting aesthetic concepts.

Resonance frequency analysis (RFA) was recorded using an Osstell ${ }^{\circledR}$ transducer (Gothenburg, Sweden) with an ISQ greater than 61 , which indicated good bone/implant contact .

Postoperative intraoral periapical radiograph was taken, confirming the accuracy of placement of implant. Abutment were attached to the implant body and postoperative laser therapy was carried out daily for 7 days. Provisional crown done with laboratory fabricated acrylic crown fixed to the abutment using light-curing composite. Temporary crown was relieved from occlusion so that they were free of protusive and lateroprotrusive contacts, which might result hat would complicate osseointegration (Figure 2A,B,C).
Appropriate antibiotic and analgesic were prescribed, and standard postoperative instructions were given to the patient. After 3 months, provisional crown were removed and impression was made with closed tray technique. Impression was sent to the laboratory for fabrication crowon. A porcelain fused to metal crowon was fabricated and cemented to the abutments Clinical and radiological observations after 3, 6 months, 1 year, 5 years showed a good clinical and aesthetic effect (Figure 3A,B,C,D).

\section{Case report 2}

A 46-year-old male patient was reported to the Department of Oral Surgery, with complaints multiple missing teeth and teeth roots. He was unsatisfied with the esthetic aspects and masticatory function.

Radiographic examination of teeth roots revealed poor prognosis. Upon intraoral examination, partially teeth loss, remaining tooth roots (Figure $4 \mathrm{~A}, \mathrm{~B}$ ).

The treatment plan included extraction 13, 1123 teeth roots immediate placement of 3 implant and insert 1 implant in position 22 tooth and 4 months after the surgery implant prosthetic rehabilitation.

Presurgical radiographic evaluation was done and appropriate length and width of available bone were determined and dental implant were selected for insertion. Under local anesthesia Sol.Ultracaini DS $4 \mathrm{ml}$, an incision was made, the
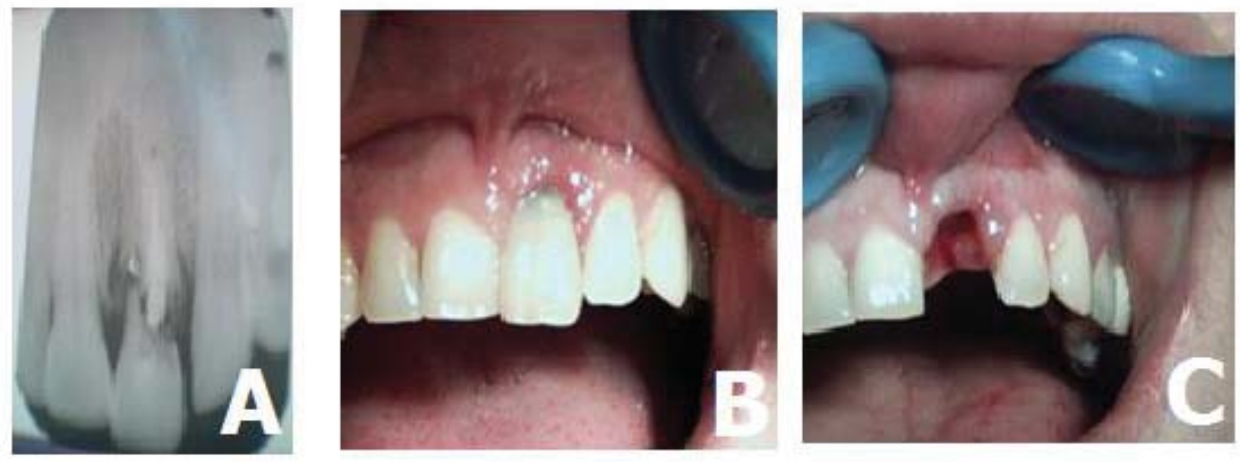

Figure 1: Radiographic and clinical examination of 12 tooth revealed poor prognosis $(A, B)$. The tooth were atraumatically removed (C).
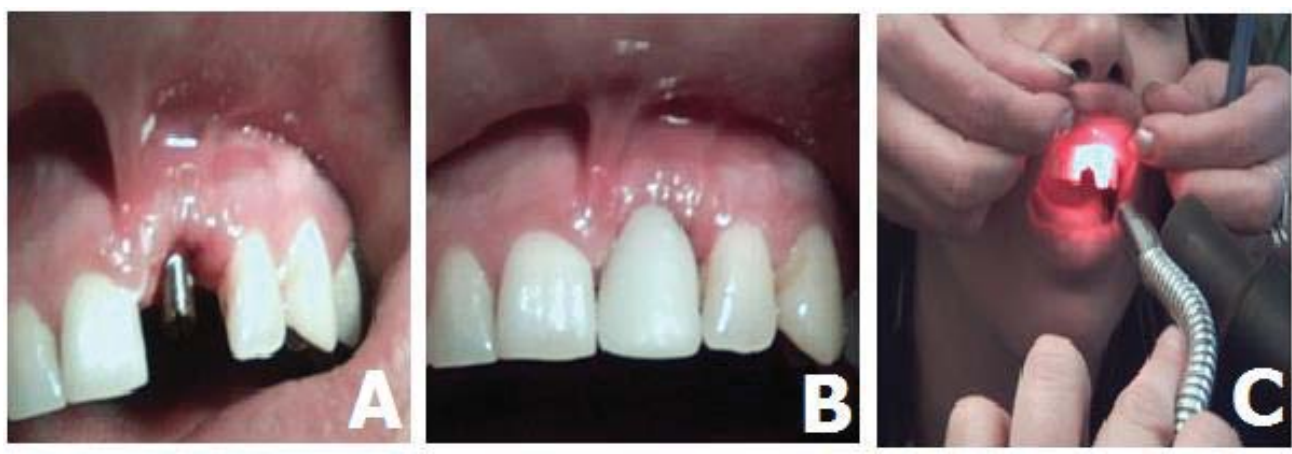

Figure 2: Abutment were attached to the implant body and provisional crown fixed to the abutment, postoperative laser therapy was carried out daily for 7 days

Citation: Hakobyan G, Esayan L, Hakobyan D, Khachatryan G, Tunyan G (2020) The comparative assessment of the of the effectiveness of immediate and delayed dental implantation. Int J Oral Craniofac Sci 6(2): 030-037. DOI: https://dx.doi.org/10.17352/2455-4634.000048 
mucoperiosteal flap was peeled, the 13, 11, 23 teeth roots were atraumatically removed. Extraction socket were thoroughly debrided and inspected with the help of periodontal probe for any defect or possible perforation of cortical plate. Osteotomy sites were prepared with sequential drills, a more palatal positioning of the implants allows a better primary stability in addition to buccal bone preservation. A osteotomysites 13 $\mathrm{mm}$ high was formed in the area of the 22 teeth. 3 implants $(4,2 \times 13 \mathrm{~mm})$, were inserted in the extraction socket and 1 inprepared osteotomy site with insertion torque of $40 \mathrm{~N} /$ $\mathrm{cm}^{2}$, and adequate primary stability was obtained. After that stage the cervical part of the installed implants was filled with a biomaterials. Resonance frequency analysis (RFA) was recorded using an Osstell ${ }^{\circledR}$ transducer (Gothenburg, Sweden) with an ISQ greater than 62, which indicated good bone/ implant contact (Figure 4.C,D,E).

The implant was closed with a heling screw and after mobilization of the mucosal-periosteal flap, sutures were placed (Figure 5A).

After implantation, prophylactic anti-inflammatory therapy was carried out for 7 days, including medications (ceflexin $500 \mathrm{mg} 2$ times a day, rinse antiseptic solution) and helium-neon laser therapy. The sutures were removed 7 days after implantation and manufacturing temporary partial denture upper jaw.

The second stage of implantation was started after 5 months. Postoperative radiograph was taken, confirming the accuracy of placement of implant (Figure 5B).
Resonance frequency analysis (RFA) was recorded using an Osstell ${ }^{\circledR}$ transducer (Gothenburg, Sweden) with an ISQ greater than 68.The orthopedic stage is completed 15 days after the second surgical stage. Abutments were attached to the implant body and prepared for parallelism and adequate space(Fig. 5C). The dental defect is restored by a metal-ceramic construction. The patient regularly appeared for follow-up examinations, 2 times a year. Clinical and radiological observations after 3, 6 months, 1 year, 5 years showed a good clinical and aesthetic effect. During subsequent follow-up examinations, the situation did not change locally (Figure 5D).

\section{Discussion}

Over the years, many solutions have been proposed in order to improve the clinical performance of dental implants [2].

In The Fourth ITI Consensus Conference (November 2009), the advantages and drawbacks of the various points in time for implant placement after tooth extraction were reported. They concluded that immediate implant placement is a more difficult technique than delayed implant placement to allow initial stability and a good prosthetic position. There is also an in-creased risk of mucosal recession. Nonetheless, based on the aesthetic index, $80 \%$ of immediate implant sites show satisfactory outcomes. The survival rates for immediate implants are high and comparable to those of implants placed in healing sites [14]. Over time, clinical experience has provided the criteria for immediate implant treatment success: atraumatic tooth extraction, minimal invasive surgical approach, as well as implant primary stability [15].
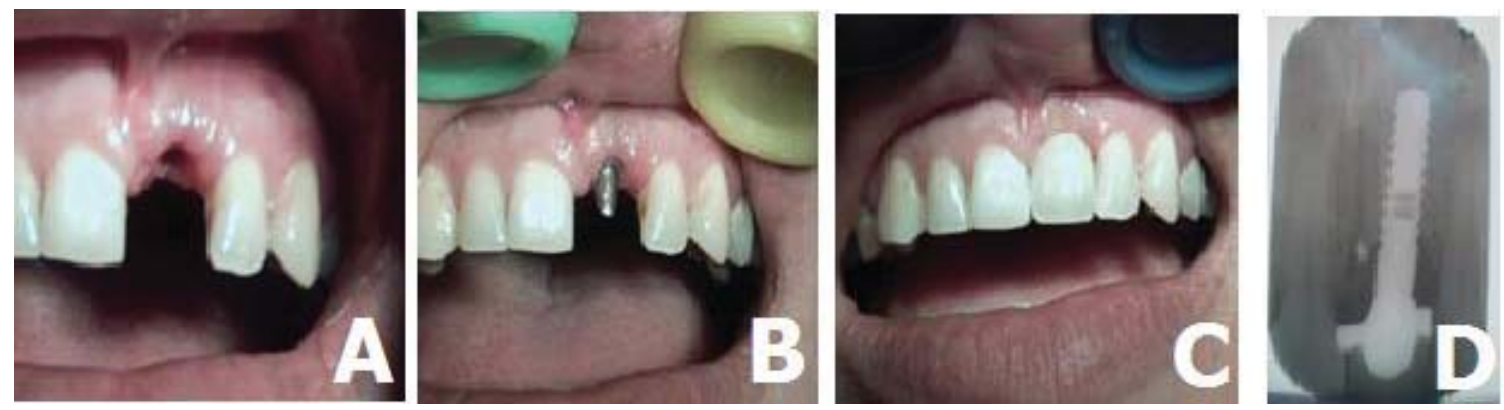

Figure 3: After 3 months, provisional crown were removed, abutment were attached to the implant body and porcelain fused to metal crowon was cemented to the abutments, clinical and radiological observations after 3 months, showed a good clinical and aesthetic effect $(A, B, C, D)$
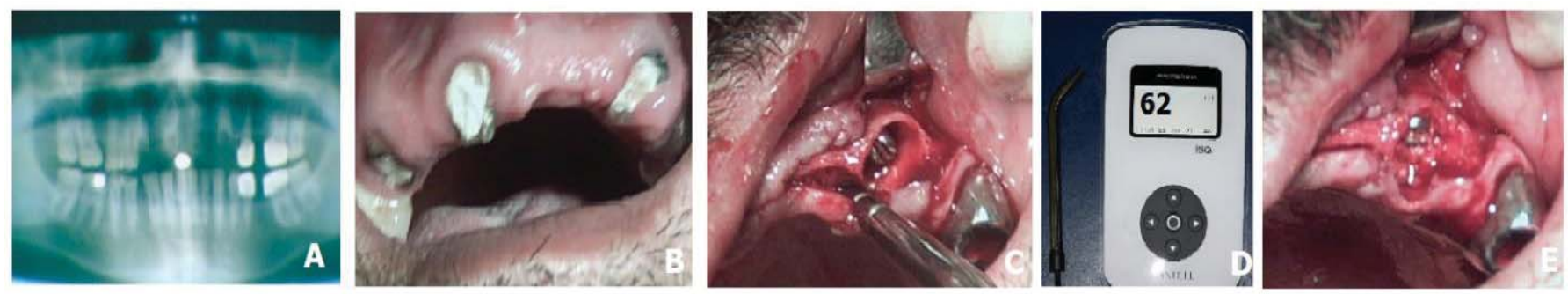

Figure 4: Radiographic examination of teeth roots revealed poor prognosis. Upon intraoral examination, partially teeth loss, remaining tooth roots (A,B), 1 implants (4,2x 13mm), were inserted in the 23 extraction socket with insertion torque of $40 \mathrm{~N} / \mathrm{cm} 2$, (C), Resonance Frequency Analysis (RFA) was recorded with an ISQ greater than 62(D), the cervical part of the installed implants was filled with a biomaterials $(\mathrm{E})$. 

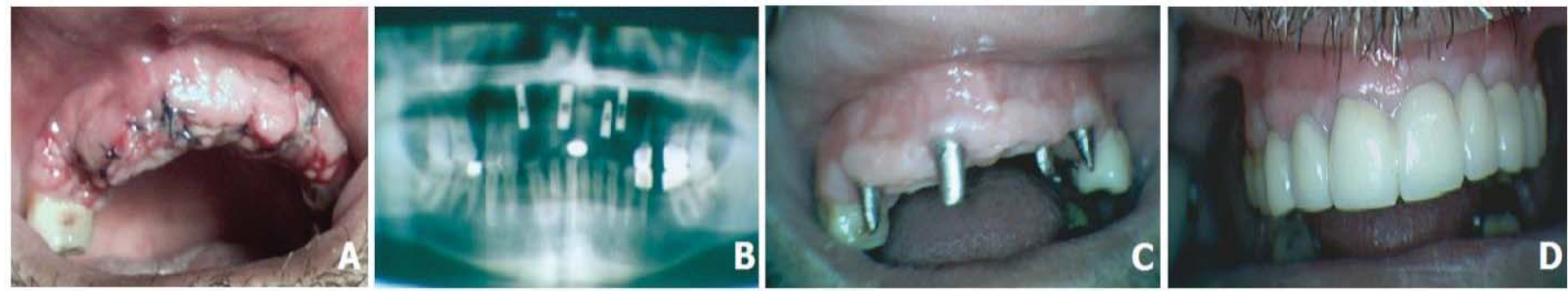

Figure 5: The implant was closed with a heling screw and after mobilization of the mucosal-periosteal flap, sutures were placed (A). Postoperative radiograph was taken confirming the accuracy of placement of implant (B). Abutments were attached to the implant body and prepared for parallelism and adequate space(C). The dental defect is restored by a metal-ceramic construction (D).

In some cases with bone defects, bone grafts are necessary, achieving good aesthetic results is difficult, as the gingival architecture and soft tissue harmony are not preserved [16].

For an immediate implant placement the most critical part of the surgery is an atraumatic tooth removal especially in the esthetic zone. Reducing buccal bone resorption are important considerations when contemplating immediate implant placement.The amount of osseous resorption is usually greater over the buccal aspect of roots than palatal and interproximally. In the first 6 to 12 months after extraction, buccal bone resorption is progressive if no bone regeneration procedure is adopted, even when there is no excessive trauma during surgery [17]. Several studies have suggested that small gaps between implants and extraction sockets would fill with bone grafting procedures or without them. With regard to the gap between the socket wall and the implant, it was reported that if the jumping distance is over $2 \mathrm{~mm}$, grafting is recommended. Smaller distances could heal spontaneously [18-20].

The esthetics of the implant get enhanced when it is placed palatally and $3 \mathrm{~mm}$ to $4 \mathrm{~mm}$ apical to the free gingival margin. In the gap between the implant and buccal bone, to graft the bone and tissue zones, autogenous, allograft, xenograft, and synthetic bone substitutes and/or materials can be used. This method can maximally leave the thin buccal wall undamaged. The graft material acts as a scaffold which maintains the blood clot for initial healing and the hard and soft tissue volume $[21,22]$.

Primary implant stability is an important factor in achieving osseointegration.There are several methods described to measure this parameter. The most common are: during the implant placement with the insertion torque, and Resonance Frequency Analysis (RFA) with the Ostell Mentor device [2325].

The benefits of immediate implant placement include fewer surgical interventions, reduced overall treatment time, reduced soft and hard tissue loss, immediate restorations can be provided for better esthetics and patient psychological satisfaction.

Nevertheless, some guidelines could be extracted from this work.

This study focused to evaluate the effectiveness and comparison immediate and delayed implant placment protocols.

With the simultaneous addition of soft- and hard-tissue grafts, the peri-implant horizontal tissue topography can be maintained which ensures the functional and aesthetic success of immediate implantation.

In our cases a void maintained between the implant and buccal wall was grafted with osteoplastic materials, which provided to slightly increase of the alveolar bone and we decided not to perform an adjunctive connective tissue graft.

Chronic periapical infection is a risk factor but not an absolute contraindication for immediate implant placement. However, debridement of the alveolus should be made. The presence of a periapical infection should be carefully weighed.

In this report, implant primary stability $\left(40 \mathrm{~N} / \mathrm{cm}^{2}\right)$ was achieved by osteotomy $3 \mathrm{~mm}$ per apex of the extraction soket and selecting an implant diametr of $0.5 \mathrm{~mm}$ wider than the width of the extraction socket. To quantify this parameters torque values and resonance frequency analysis (RFA) Methods were used. There are no significant differences between primary stability of immediate and delayed implants, but in both cases implant stability increases during the healing process.

Immediate implant placement with immediate loading may be a treatment option for cases requiring earliest restoration of teeth to be extracted, long as all criteria for initial implant stability and occlusal adjustment of the provisional restoration are met. The immediate loading implant placement preserves the vertical existing osseous and maintains the gingival architecture.

There are no significant differences in immediate and delayed implants. After dental implant prosthetic rehabilitation, the masticatory function, esthetics of the facial profile and occlusion was improved. Patients expressed satisfaction with the result of treatment and improved quality of life.

Careful selection of cases, proper treatment plan and follow-up of surgical and prosthetic protocols are the keys to success.The immediate implant placement saves time, involve less invasive surgical procedures, the minimal soft tissue and crestal bone loss, and considerably very good esthetic outcome and excellent success rates. 
Based on the results of this report it can be concluded that immediate placement of the implant may be a favorable treatment option if there is sufficient keratinized gingival tissuesthickness and sufficient bone volume in the area of the extracted tooth, the absence of acute inflammation in the well of the extracted tooth socket and good primary fixation of the implant in extraction socket.

\section{Conclusion}

Based on this, immediate implant placement following tooth extraction might be a alternative to delayed implant placement. Immediate placement of the implant prevents atrophy of the alveolar ridge thereby preventing recession of the mucosal and gingival tissues and can be provided better esthetics. However, immediate implant placement requires a careful case selection and a specific treatment protocol because it is a more difficult to execute than a conventional protocol.

\section{Consent statement}

Written informed consent was obtained from the patient for publication of this case report and accompanying images.

\section{References}

1. Meyer MS, Joshipura K, Giovannucci E, Michaud DS (2008) A review of the relationship between tooth loss, periodontal disease, and cancer. Cancer Causes Control 19: 895-907. Link: https://bit.ly/2ljSMHI

2. Desvarieux M, Demmer RT, Rundek T, Boden-Albala B, Jacobs DR, et al (2003) Relationship between periodontal disease, tooth loss, and carotid artery plaque: The oral infections and vascular disease epidemiology study (INVEST). Stroke 34: 2120-2125. Link: https://bit.ly/2ljTgNr

3. Anil S, Preethanath RS, AlMoharib HS, Kamath KP, Anand PS (2013) Impact of osteoporosis and its treatment on oral health. Am J Med Sci 346: 396401. Link: https://bit.ly/36lwyfV

4. Craddock HL (2009) Consequences of tooth loss: 1. The patient perspective - Aesthetic and functional implications. Dent Update 36: 616-619. Link: https://bit.ly/2U8siL9

5. Schropp L, Isidor F (2008) Timing of implant placement relative to tooth extraction. JOral Rehabil 35: 33-43. Link: https://bit.ly/38qogGz

6. Schulte W, Heimke G (1976) The Tubinger immediate implant. Quintessenz 27 17-23. Link: https://bit.ly/3n41HLK

7. Douglass GL, Merin RL (2002) The immediate dental implant. J California Dent Assoc 30: 362-365. Link: https://bit.ly/2JQJ0Nn

8. Chen S, Wilson TG, Hämmerle $\mathrm{CH}$ (2004) Immediate or early placement of implants following tooth extraction: review of biologic basis, clinical procedures, and outcomes. Int J Oral Maxillofac Implants 19: 12-25. Link: https://bit.ly/2JI4xrk

9. Chen CL, Chang CL, Lin SJ (2011) Immediate implant placement and provisionalization with simultaneous guided bone regeneration in the esthetic zone. J Dent Sci 6: 53-60. Link: https://bit.ly/3lcr0e0

10. Singh A, Gupta A, Yadav A, Chaturvedi TP, Bhatnagar A, et al. (2012) Immediate placement of implant in fresh extraction socket with early loading. Contemp Clin Dent 3: 219-222. Link: https://bit.ly/36lx8KD
11. Singh M, Kumar L, Anwar M, Chand $P$ (2015) Immediate dental implant placement with immediate loading following extraction of natural teeth. Natl $J$ Maxillofac Surg 6: 252-255. Link: https://bit.ly/3p5iCiO

12. Quirynen M, Van Assche N, Botticelli D, Berglundh T (2007) How does the timing of implant placement to extraction affect outcome? Int J Oral Maxillofac Implants 22: 203-223. Link: https://bit.ly/3pbdyto

13. Ferrara A, Galli C, Mauro G, Macaluso GM (2006) Immediate provisional restoration of postextraction implants for maxillary single-tooth replacement. Int J Periodontics Restorative Dent 26: 371-377. Link: https://bit.ly/2U61jQq

14. Chen ST, Beagle J, Jensen SS, Chiapasco M, Darby I (2009) Consensus statements and recommended clinical procedures regarding surgical techniques. Int J Oral Maxillofac Implants 24: 272-278. Link: https://bit.ly/3eEDnNw

15. Siegenthaler DW, Jung RE, Holderegger C, Roos M, Hämmerle CH (2007) Replacement of teeth exhibiting periapical pathology by immediate implants: A prospective, controlled clinical trial. Clin Oral Implants Res 18: 727-737. Link: https://bit.ly/3kbbrlG

16. Kois JC (2004) Predictable single-tooth peri-implant esthetics: five diagnostic keys. Compend Contin Educ Dent 25: 595-596.

17. Araújo MG, Lindhe J (2005) Dimensional ridge alterations following tooth extraction. An experimental study in the dog. J Clin Periodontol 32: 212-218. Link: https://bit.ly/36ygJTB

18. Covani U, Cornelini R, Barone A (2008) Buccal bone augmentation around immediate implants with and without flap elevation: a modified approach. Int J Oral Maxillofac Implants 23: 841-846. Link: https://bit.ly/3kbcn9G

19. Gherlone E, Romanos GE (2007) Immediate occlusal loading of implants placed in fresh sockets after tooth extraction. Int J Oral Maxillofac Implants 22: 955-962. Link: https://bit.ly/32scyaw

20. Chen CL, Chang CL, Lin SJ (2011) Immediate implant placement and provisionalization with simultaneous guided bone regeneration in the esthetic zone. J Dent Sci 6: 53-60. Link: https://bit.ly/3lcr0e0

21. Lang NP, Tonetti MS, Suvan JE, Pierre Bernard J, Botticelli D, et al. (2007) Immediate implant placement with transmucosal healing in areas of aesthetic priority. A multicentrerandomizedcontrolled clinical trial I. Surgical outcomes. Clin Oral Implants Res 18: 188-196. Link: https://bit.ly/2UmhiKx

22. Sanz M, Cecchinato D, Ferrus J, Pjetursson EB, Lang NP, et al. (2010) A prospective, randomized-controlled clinical trial to evaluate bone preservation using implants with different geometry placed into extraction sockets in the maxilla. Clin Oral Implants Res 21: 13-22. Link: https://bit.ly/3eEjzKj

23. Vidyadharan A, Hanawa Y, Godfrey S, Resmi PG (2014) Immediate implants and immediate loading in full arch maxilla and mandible of a bruxerA case report. IOSR J Dent Med Sci 13: 62-67. Link: https://bit.ly/35cvSdH

24. Park JC, Lee JW, Kim SM, Lee JH (2011) Implant Stability - Measuring Devices and Randomized Clinical Trial for ISQ Value Change Pattern Measured from Two Different Directions by Magnetic RFA. Rapidly Evolving Practice 5: 111 130. Link: https://bit.ly/3khKvQZ

25. Valderrama P, Oates TW, Jones AA, Simpson J, Schoolfield JD, et al. (2007) Evaluation of two different resonance frequency devices to detect implant stability: A clinical trial. J Periodontol 78: 262-272. Link: https://bit.ly/38DoWZ

26. Dario LJ, Cucchiaro PJ, Deluzio AJ (2002) Electronic monitoring of dental implant osseointegration. J Am Dent Assoc 133: 483-490. Link: https://bit.ly/36neamY

Copyright: @ 2020 Hakobyan G, et al. This is an open-access article distributed under the terms of the Creative Commons Attribution License, which permits unrestricted use, distribution, and reproduction in any medium, provided the original author and source are credited.

Citation: Hakobyan G, Esayan L, Hakobyan D, Khachatryan G, Tunyan G (2020) The comparative assessment of the of the effectiveness of immediate and delayed dental implantation. Int J Oral Craniofac Sci 6(2): 030-037. DOI: https://dx.doi.org/10.17352/2455-4634.000048 